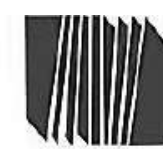

UNIVLRSIDADPEDAGOGICA EXPLR IALNTAL LIELRTROOK

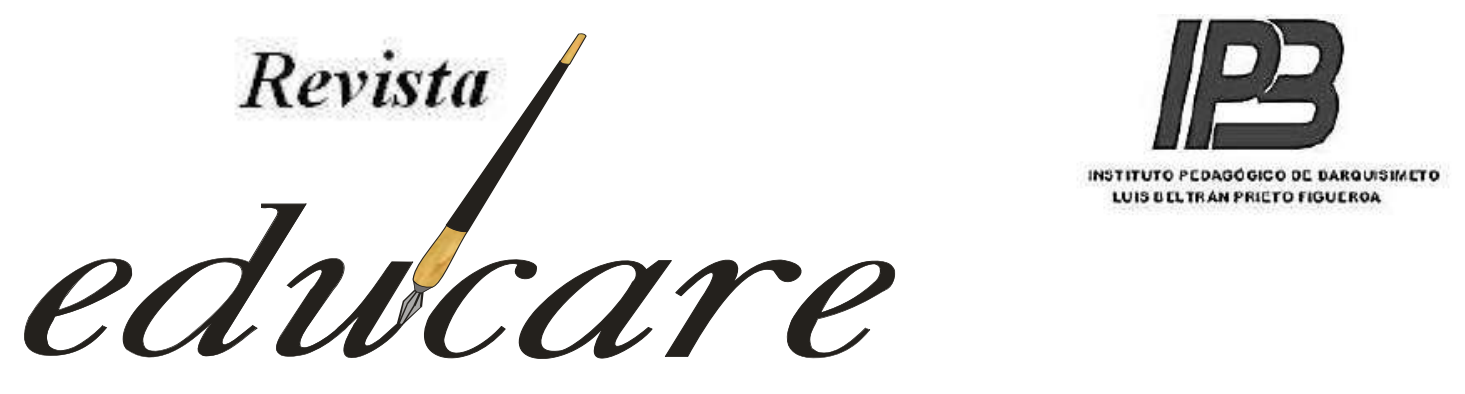

Órgano Divulgativo de la Subdirección de Investigación y Postgrado del Instituto Pedagógico de Barquisimeto "Luis Beltrán Prieto

Figueroa"

BARQUISUUEIO - EDO. LARA - VEREZUEA

\title{
MODELO ATÓMICO: SIMULACIÓN DE LAS PRINCIPALES EXPERIENCIAS QUE LLEVARON A SU FORMULACIÓN
}

\author{
ATOMIC MODEL: SIMULATION OF THE MAIN EXPERIENCES THAT GENERATED \\ ITS FORMULATION
}

\section{DEPOSITO LEGAL: ppi201002LA3674 ISSN: 2244-7296}

Sergio Baggio *

\section{Universidad Nacional de la Patagonia San Juan Bosco, Puerto Madryn, Argentina}




\begin{tabular}{|c|c|}
\hline PROPUESTA EDUCATIVA & $\begin{array}{l}\text { Sergio Baggio }^{*} \\
\text { UTN-FRCh, UNPSJB }\end{array}$ \\
\hline Recibido: 18-01-2016 & Aceptado: $30 / 05 / 2016$ \\
\hline RESUMEN & ABSTRACT \\
\hline $\begin{array}{l}\text { El objetivo del presente trabajo es la } \\
\text { presentación y descripción de un conjunto de } \\
\text { programas de simulación, desarrollados por el } \\
\text { autor, para ser utilizados en el proceso de } \\
\text { enseñanza-aprendizaje del tema formulación del } \\
\text { modelo atómico, tanto para la educación básica } \\
\text { universitaria como para la educación media. Se } \\
\text { trata de una propuesta educativa que apunta a } \\
\text { resolver algunos de los problemas que se } \\
\text { presentan en el estudio de este tema. La } \\
\text { experimentación es fundamental para el } \\
\text { aprendizaje de las ciencias fácticas y la química } \\
\text { no escapa a esta afirmación. En el tema modelo } \\
\text { atómico el costo del equipamiento para realizar } \\
\text { estos experimentos, hacen que sea poco factible } \\
\text { realizarlas en nuestras escuelas y universidades, } \\
\text { que generalmente carecen de los mismos. Por ello } \\
\text { se propone el uso de simulaciones como una } \\
\text { interesante alternativa para su estudio. Con estos } \\
\text { programas el docente puede introducir al alumno } \\
\text { en un tema que es esencialmente abstracto a } \\
\text { través de experiencias "concretas" y siguiendo un } \\
\text { enfoque de descubrimiento guiado. } \\
\text { Descriptores: Simulaciones, modelo atómico, } \\
\text { química general. }\end{array}$ & $\begin{array}{l}\text { The objective of the present work is the } \\
\text { presentation and description of a set of } \\
\text { simulation programs, developed by the author, } \\
\text { to be used in the teaching-learning process of } \\
\text { the formulation of the atomic model, for both } \\
\text { basic university education and high school. It is } \\
\text { an educational proposal that aims to solve some } \\
\text { of the problems that are presented in the study } \\
\text { of this topic. Experimentation is fundamental to } \\
\text { the learning of the factual sciences and } \\
\text { chemistry does not escape to this statement. In } \\
\text { the atomic model topic the cost of the } \\
\text { equipment to carry out these experiments, } \\
\text { makes it impractical to perform them in our } \\
\text { schools and universities, due to the lack of the } \\
\text { equipments. Therefore, the use of simulations as } \\
\text { an interesting alternative for its study is } \\
\text { proposed. With these programs the teacher can } \\
\text { introduce the student into a subject that is } \\
\text { essentially abstract through "concrete" } \\
\text { experiences and following a guided discovery } \\
\text { approach. }\end{array}$ \\
\hline
\end{tabular}

\footnotetext{
*Dr. de la Universidad de Buenos Aires, Profesor Titular Consulto, Universidad Tecnológica Nacional Facultad Regional Chubut, Universidad Nacional de la Patagonia San Juan Bosco, Puerto Madryn, Argentina. baggiosergio@yahoo.com.ar--
} 


\section{INTRODUCCIÓN}

La formulación del modelo atómico está íntimamente ligado a la vinculación que se fue estableciendo entre la materia y la electricidad, a través de las primeras experiencias realizadas por Davy en 1800, cuando encontró que ciertos compuestos se descomponían por el paso de la corriente eléctrica y sus componentes podían ser depositados sobre los electrodos. Estos trabajos fueron cuantificados luego por Faraday en 1833 al relacionar la masa y la cantidad de electricidad puestas en juego, lo que sugería claramente la naturaleza eléctrica de la materia.

En la segunda mitad del siglo XIX comienzan las experiencias con tubos de descarga, destacándose Thomson quien encontró que cuando se aplica un alto voltaje a través de electrodos en un recipiente de vidrio del que se ha extraído la mayor parte del aire, se produce una descarga eléctrica que proviene del cátodo generando los llamados rayos catódicos. Estos viajan en líneas rectas, ya que proyectan una sombra sobre una pantalla fluorescente, cuando se interpone un objeto en su camino. Además, estos rayos pueden ser desviados por los campos magnéticos y eléctricos, sugiriendo que están constituidos por cargas eléctricas en movimiento. La dirección de la deflexión de la carga de estas partículas confirmó que la misma era de signo negativo. En 1897 Thomson midió por primera vez la relación entre la carga y la masa(e/m), de estas pequeñas partículas, bautizadas como electrones por Stoney en 1874.

La determinación de la carga del electrón a través de la famosa experiencia de la gota de aceite de Millikan, terminó de caracterizar a esta partícula al conocerse su carga y su masa (Pearson, 2005).

El descubrimiento de los Rayos X por parte de Roentgen (Garrett, 1962a) y de la radiactividad por Becquerel (Garrett, 1962b) aportaron nuevos elementos para el avance del modelo.

La experiencia determinante es probablemente la que realizaron a principios del siglo XX Rutherford, Geiger y Marsden, en 1911 (Herron, 1977), bombardeando una delgada lámina de oro con partículas alfa. Se sabía que las partículas alfa, recientemente descubiertas, eran iones de helio que viajaban en líneas rectas a velocidades muy altas. El modelo atómico que sugería Thomson para el átomo, como una esfera de materia positiva 
en la que los electrones se incorporaban al azar (como las pasas en un pastel), sugería que las partículas alfa pasarían sin dificultad a través de la lámina metálica.

Sin embargo los resultados obtenidos fueron sorprendentes, con algunas partículas que prácticamente rebotaban en la lámina de oro, lo que llevó a Rutherford a postular su modelo del átomo como algo esencialmente hueco con un núcleo muy pequeño que contiene casi toda la masa y la carga positiva y los electrones de carga negativa distribuidos alrededor de ese núcleo.

El protón, que así se llamó a la unidad de carga positiva, y que fuera detectado por primera vez en el estudio de los rayos canales, tenía una masa aproximadamente 1836 veces mayor a la del electrón y no daba cuenta de toda la masa que tenía el átomo. Por ello se postuló la existencia del neutrón en el núcleo, una partícula sin carga pero con masa semejante a la del protón y que recién fue descubierta por Chadwick en 1932 (Peake, 1989).

La distribución de los electrones alrededor del núcleo, fundamental para el estudio de las propiedades químicas de los elementos, comienza a delinearse con Bohr para luego ser completada con el desarrollo de la mecánica cuántica.

\section{ARGUMENTACIÓN TEÓRICA}

El desarrollo del modelo atómico, forma parte, con mayor o menor profundidad de cualquier curso de química básica ya que es fundamental para el estudio posterior de la asignatura, especialmente en los temas del enlace químico y las propiedades de los elementos y compuestos. Surge la pregunta de cuál es la mejor manera de encarar el estudio de este tema, por tratarse de contenidos mayoritariamente abstractos.

Hay un debate aun abierto sobre cuál es el mejor enfoque para el proceso de enseñanza-aprendizaje en ciencia. La pregunta es: ¿Quién debe liderar el proceso, el docente o el alumno (Tobias y Duffy, 2009)? Las posiciones adoptadas varían desde una preferencia por los métodos expositivos dirigido por el docente (Kirschner, Sweller y Clark, 2006) o los enfoques totalmente abiertos, llamados generalmente de descubrimiento puro (Papert, 1980) con posiciones intermedias representadas por los procesos de 
descubrimiento más o menos guiados (Mayer, 2004), al cual adherimos en el enfoque de este trabajo.

Al discutir la cuestión del papel de la orientación en la enseñanza, la tecnología específica de simulaciones por ordenador ocupa un lugar central. Las simulaciones por ordenador, a través de su carácter interactivo, ofrecen una oportunidad especial para el aprendizaje centrado en el estudiante, aunque al mismo tiempo ofrece opciones para el otro enfoque, o sea el liderado por el docente.

En particular, el dictado que realizamos en la asignatura de Química General en la UNPSJB está orientado a cursos que tienen un desarrollo de descubrimiento guiado (Karplus, 1977; Kolb, 1984; Farrell, Spencer y Moog, 1999), con una secuencia de actividades que puede resumirse así: explorar $=>$ inventar $=>$ expandir $=>$ ejercitar . En estos cursos se propone que la primera actividad que realice el alumno sea en el laboratorio donde éste, con una mínima guía, ejecute experimentos relacionados con el tema a tratar y que sean esencialmente cualitativos o semi cuantitativos. Esto le permite realizar una observación reflexiva sobre los mismos, dejando la etapa de mediciones para las simulaciones, cuando éstas puedan ser incorporadas. En el tema que nos convoca es difícil realizar experimentación debido a la complejidad y costo de los equipos que hace que la mayoría de nuestras escuelas o universidades carezcan de los mismos.

Entonces frente a las dificultades para experimentar con los alumnos principiantes y ante un tema tan abstracto como es el desarrollo del modelo atómico, las simulaciones se presentan como una interesante alternativa para poder realizar la secuencia de descubrimiento guiado antes mencionado (Baggio, 2009). Una busqueda en la Web de programas de simulación en el tema de la estructura atómica, muestra muchos resultados positivos. Sin embargo, la mayoría de los programas encontrados se encuentran en un idioma que no es el español, tienen un costo y no siempre siguen un desarrollo de investigación guiada, que es al que adherimos.

\section{DESCRIPCIÓN DE LA PROPUESTA Y ASPECTOS METODOLÓGICOS}

A fin de realizar esta actividad se han desarrollado once programas de simulación que cubren los aspectos más salientes de la formulación del modelo atómico. Una simulación 
utiliza un modelo matemático o lógico para recrear una situación o fenómeno. El sistema o proceso que se modela normalmente tiene un origen en el mundo natural y el modelo que se crea es por lo general una simplificación del mismo (de Jong, 2011).

La simplificación se usa porque: (1) es difícil, si no imposible modelar totalmente el mundo real; (2) la simplificación, a menudo, es suficiente para lograr el objetivo para el que se construye el modelo y un mayor realismo también tiene costos adicionales en tiempo, recursos y esfuerzo; y (3) las simplificaciones producen menor carga cognitiva para el alumno y eso facilita generalmente el aprendizaje.

Permite al estudiante controlar la interactividad de los elementos dinámicos que están siendo estudiados (Rieber, 2005). Así el alumno (por ejemplo, en el tema del tubo de rayos catódicos) puede realizar una acción (variables independientes) como aplicar un campo eléctrico o magnético, para establecer un resultado, que debe medir y que es la variable dependiente (la desviación que sufre el haz de rayos catódicos por esta acción).

El resultado es, pues, una consecuencia de sus decisiones, que puede ser representado como un resultado verbal o matemático, una visualización estática, o una visualización dinámica. Los objetivos de una simulación incluyen: (a) ayudar a los estudiantes a estimar la probabilidad de varios resultados; (b) centrar su atención en una parte de la realidad a expensas de las otras partes; o (c) ayudar a apreciar cómo funciona un sistema cuando se cambian los valores de entrada para cada variable independiente. La combinación de estos tres aspectos permite a los estudiantes hacer predicciones sobre el comportamiento del sistema y recibir información acerca de cómo funciona el mismo.

Los temas abordados en las simulaciones desarrolladas con una breve descripción de sus alcances, son:

1) Rayos catódicos

Se simula la experiencia de generación de rayos catódicos. Se estudia la respuesta de los mismos frente a la aplicación de campos eléctricos o magnéticos. Se determina la relación carga/masa del electrón (el funcionamiento se ilustra en detalle más adelante).

2) Experiencia de Millikan

Se simula la experiencia de Millikan para determinar la carga del electrón. Se visualiza a través de un anteojo una gotita de aceite proveniente de un rocío y se mide su velocidad de caída, que es constante. Luego rápidamente se aplica un campo eléctrico para 
detenerla en su caída. Con los datos recogidos de velocidad y campo eléctrico aplicado se calcula la carga que lleva la partícula. Se repite el proceso con varias gotitas. El máximo común divisor de todos los valores obtenidos representa la carga del electrón (se ilustra en detalle más adelante).

3) Radiactividad

Se simulan algunas propiedades de las radiaciones generadas en los procesos radiactivos. Se estudian tres isótopos radiactivos ${ }_{6}^{14} \mathrm{C},{ }_{27}^{60} \mathrm{Co}$ y ${ }_{88}^{226} \mathrm{Ra}$, emisores de diferentes radiaciones. Se analizan las radiaciones emitidas aplicando un campo eléctrico. Los procesos que se producen en la desintegración son

$$
\begin{gathered}
{ }_{6}^{14} \mathrm{C} \rightarrow{ }_{7}^{14} \mathrm{~N}+\beta \\
{ }_{27}{ }_{27} \mathrm{Co} \rightarrow{ }^{60}{ }_{27} \mathrm{Co}+\gamma \\
{ }^{226}{ }_{88} \mathrm{Ra} \rightarrow{ }^{222}{ }_{86} \mathrm{Rn}+\alpha
\end{gathered}
$$

por lo que al aplicar el campo eléctrico dos de las radiaciones se desvían en direcciones opuestas y una no sufre desviación.

4) Experiencia de Rutherford

Se simula la experiencia de la lámina de oro de Rutherford. Se observan los destellos que produce el impacto de las partículas alfa sobre una pantalla fluorescente y al final se muestra, en forma relativa, el número de impactos en función del ángulo de desviación (Mahan y Myers, 1987).

\section{5) Rayos X característicos}

Se simula la generación de los rayos $\mathrm{X}$ característicos que desemboca en la postulación del número atómico (Haigh, 1995). Se dispone de un tubo de rayos X con ánodo intercambiable a fin de utilizar en él distintos metales. La radiación emitida por el tubo, una vez colimada, se hace incidir sobre un cristal analizador que rota y cuando el ángulo es tal que se cumple la ley de Bragg (Atkins y Jones, 1997) aparece una línea sobre una pantalla fluorescente.

Una vez barridos todos los ángulos permitidos, se miden las longitudes de onda registradas y se propone al alumno que encuentre alguna relación vinculada con el elemento metálico del ánodo. Debería aparecer el concepto del número atómico. Esto da pie para resaltar las incongruencias en la tabla de Mendeleev respecto de las inversiones en 
los pares $(\mathrm{Ni}, \mathrm{Co}),(\mathrm{Ar}, \mathrm{Kr})$ y $(\mathrm{Te}, \mathrm{I})$. El primero de los pares mencionados se analiza en la simulación. Se puede encontrar y enunciar la ley de Moseley.

6) Espectros electrónicos

Se simula el espectro de emisión de una lámpara incandescente (luz blanca) y también la de varios elementos $\left(\mathrm{H}_{2}, \mathrm{He}\right.$ y $\left.\mathrm{Hg}\right)$. En el caso del espectro de emisión del hidrógeno se miden las longitudes de onda registradas en la región del visible y se alienta a los alumnos para que encuentren alguna relación entre los números obtenidos. Aquí es necesaria la activa participación del docente a fin de poder llegar a la relación encontrada por Balmer (Herzberg, 1944)

7) Configuración electrónica

Varios prestigiosos autores han cuestionado la introducción temprana, en los cursos básicos de química de los resultados de la Mecánica Cuántica (Pauling, 1984; Gillespie, 1991). Siguiendo los lineamientos propuestos por Gillespie y haciendo uso de los potenciales de ionización y de los espectros fotoelectrónicos se trata de construir los niveles de energía de los primeros elementos de la tabla periódica a través del modelo de capas, sin recurrir a los números cuánticos, que aparecen recién al final para darle al alumno el vocabulario que se utiliza actualmente para caracterizar a los electrones de un átomo (Moog \& Farrell, 2004). Finalmente el alumno puede ejercitar la escritura de la configuración electrónica de los elementos mencionados actuando el programa como control de la respuesta.

8) Espectro de masas

Se simula el espectro de masas de algunos elementos que conduce al “descubrimiento" de los isótopos. De la observación del espectro se puede estimar el peso atómico del elemento en base a los porcentajes detectados para cada isótopo. Es interesante analizar con los alumnos el caso del cloro, que como se sabe presenta 2 isótopos: el de número de masa 35 u y el 37 u. En el espectro no sólo se ven números de masa 70 u y 74 u, correspondiendo a las respectivas moléculas, sino también 72 u que corresponde a moléculas formadas por átomos de isótopos diferentes.

9) Efecto fotoeléctrico

En 1902 se sabía que cuando la luz incidía sobre la superficie limpia de ciertos metales en el vacío se producía la emisión de electrones. En 1905, Einstein indicó que el 
llamado efecto fotoeléctrico podía ser explicado si la luz se consideraba constituida por partículas discretas de energía $h v$ o fotones. El desarrollo de esta teoría le valió el premio Nobel de física. En el programa se simula el efecto fotoeléctrico y se miden parámetros que permiten calcular la constante de Planck $h$. Los metales que se estudian son algunos alcalinos y alcalinotérreos.

10) Descubrimiento del neutrón

En la segunda década del siglo XX, el átomo se pensaba como un núcleo masivo orbitado por electrones. Las medidas de masas atómicas sugerían que en el núcleo debía haber otras partículas además de los protones. Rutherford las pensaba como "dobletes neutros" formadas por protones y electrones fuertemente unidos. Después de los trabajos de los alemanes Bote y Becker en 1930 y los esposos Curie en 1932, Chadwick realizó los experimentos definitivos que llevaron al descubrimiento del neutrón.

En el programa, se simula cualitativamente la experiencia que llevó al descubrimiento del neutrón por Chadwick.

11) Rayos positivos

Los estudios de los rayos positivos o canales por Goldstein en 1886, fueron fundamentales para el avance del modelo ya que condujo al descubrimiento del protón (Babor y Ibarz, 1968). Se simula la experiencia del tubo de rayos catódicos con cátodo perforado que llevó al descubrimiento de los rayos canales o positivos.

Algunas de las simulaciones son esencialmente cualitativas mientras que en otras el alumno puede medir y obtener parámetros fundamentales como carga y masa de electrón, longitudes de onda de rayos $\mathrm{X}$ característicos, constante de Planck, entre otras. Dependiendo del nivel educativo donde se desempeña el docente, algunas de las simulaciones más complejas podrán ser usadas para describir solamente los aspectos cualitativos del experimento y dejar los aspectos cuantitativos para los cursos universitarios más avanzados.

A fin de tener una idea más clara sobre el alcance de los programas, se describirán en detalle 2 de ellos, el de la experiencia de los rayos catódicos y el de la experiencia de Millikan. 


\section{Simulación del tubo de rayos catódicos y la experiencia de Thomson}

Los gases no son conductores de la electricidad, pero cuando se los somete a altos voltajes y están a presiones muy bajas, aparece conducción, acompañada de emisión de luz. Cuando la presión del gas baja hasta $10^{-4}$ Atm, la conducción persiste, aunque la luminosidad disminuye. Si los voltajes aplicados son lo suficientemente altos, en la pared del vidrio que encierra al gas se produce una ligera fluorescencia en algunos lugares. En 1890 varios experimentadores atribuyeron esta luminosidad al bombardeo del vidrio por rayos que se originaban en el cátodo o polo negativo y por ello se los llamó rayos catódicos.

Una vez iniciado el programa aparece la imagen de la Figura 1 y se solicita al alumno que describa lo observado, detallando la estructura del tubo de rayos catódicos.

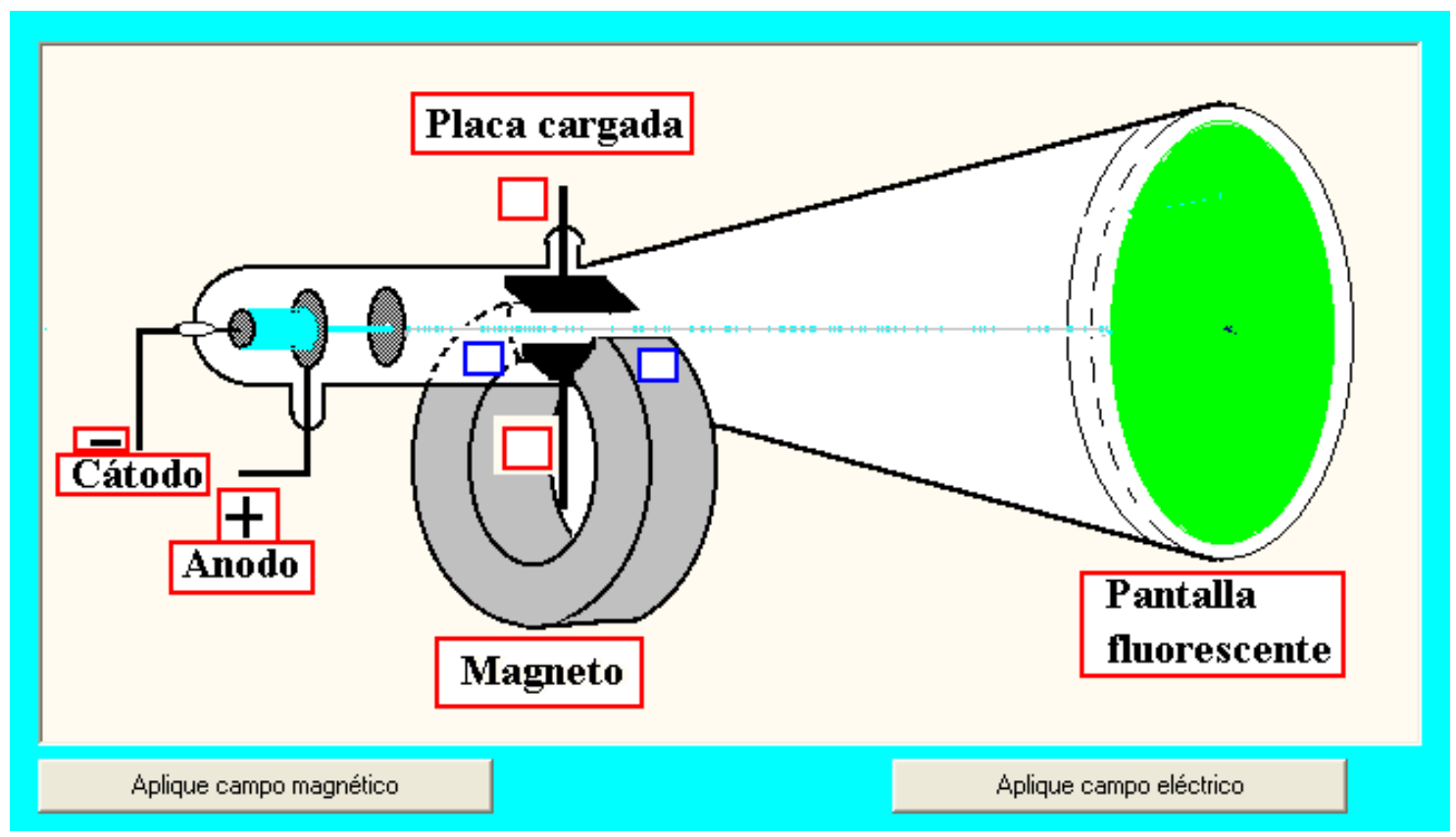

Figura 1. Esquema de un tubo de rayos catódicos.

A continuación se pulsa alguna de las teclas "Aplicar campo eléctrico" ó "Aplicar campo magnético" y se observan los resultados sobre la pantalla del tubo de rayos catódicos. Al aplicar el campo eléctrico se deben sacar conclusiones sobre la carga que llevan los rayos catódicos. Cuando se aplica el campo magnético se recuerda al alumno que la fuerza que ejerce un campo magnético sobre una carga en movimiento, está dada por la regla de la mano izquierda. Esta regla indica hacia donde está dirigida la fuerza, cuando una partícula de carga positiva se mueve en la dirección "x". El campo magnético es un vector 
cuya dirección es norte-sur. Si se trata de una partícula de carga negativa, la dirección de la fuerza es opuesta a la determinada para la carga positiva. En la Figura 2, se observa la aplicación de la regla.

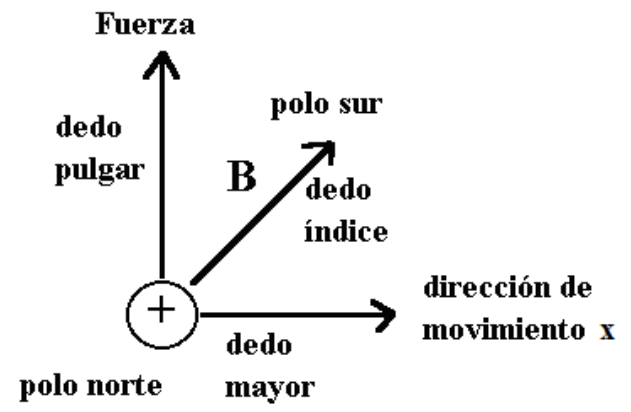

Figura 2. Aplicación de la regla de la mano izquierda.

Se realiza entonces la siguiente pregunta al alumno: ¿Están de acuerdo los resultados de la experiencia con el campo magnético con la carga que postuló anteriormente, cuando aplicó un campo eléctrico? Una vez obtenida la respuesta correcta se prosigue con la ejecución del programa.

A continuación se pulsa la tecla "Determinar la relación carga/masa". Aparece en pantalla la imagen que se muestra en la Figura 3.

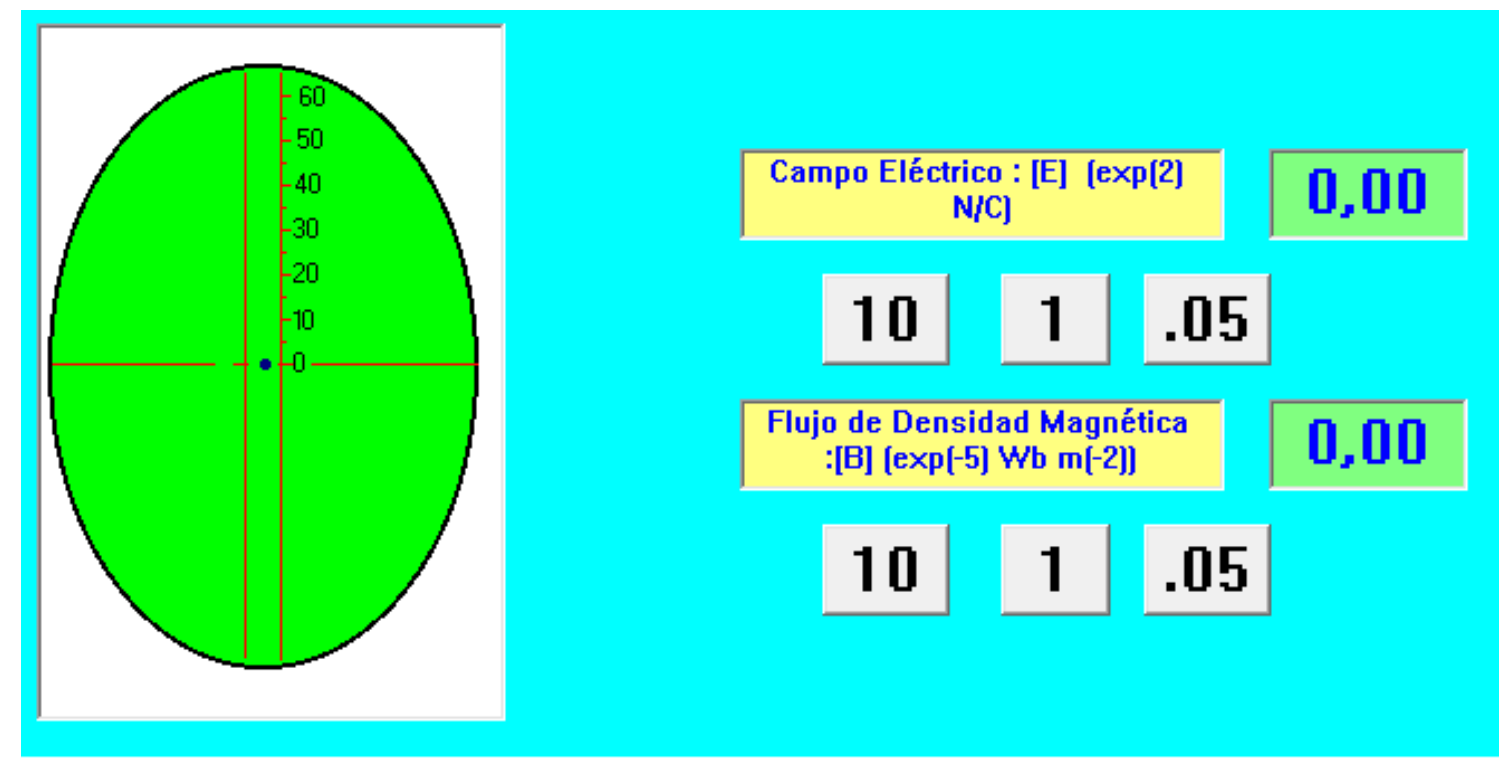

Figura 3. Pantalla inicial cuando se quiere determinar la relación e/m.

Aparecerá ampliada la pantalla fluorescente del tubo de rayos catódicos, donde se podrá apreciar la luminosidad debida al impacto de los rayos sobre la misma. Ahora se 
podrán aplicar campos eléctricos y magnéticos variables, observando los efectos que producen sobre el punto luminoso de la pantalla. Se debe recordar que la fuerza que ejerce el campo eléctrico $\mathrm{E}$ sobre una carga eléctrica $\mathrm{q}$ es $\mathrm{F}=\mathrm{q} \times \mathrm{E}$, mientras que la que ejerce el flujo de densidad magnética $\mathrm{B}$ es $\mathrm{F}=\mathrm{q} \times \mathrm{v} \times \mathrm{B}$, donde $\mathrm{v}$ es la velocidad de la partícula. Las direcciones son las que resultaron de la aplicación de la regla de la mano izquierda. Los valores de $\mathrm{E}$ que se seleccionen se deberán multiplicar por $10^{2}$ antes de realizar los cálculos, mientras que los de B por $10^{-5}$. Las unidades de E son N/C (N: Newton; C: Coulomb) mientras que las de $\mathrm{B}$ son $\mathrm{Wb} / \mathrm{m}^{2}(\mathrm{~Wb}=\mathrm{Weber})$, de esa manera las unidades de velocidad resultan en $\mathrm{m} / \mathrm{s}$.

La secuencia de operaciones es que se aplica un campo eléctrico hasta obtener una desviación razonable del haz, por ejemplo la que se muestra en la Figura 4 donde el haz se desplaza cerca de $60 \mathrm{~mm}$ por la aplicación de un campo eléctrico de $70 \times 10^{2} \mathrm{~N} / \mathrm{C}$. Se registra el valor del desplazamiento, necesario para los cálculos posteriores.

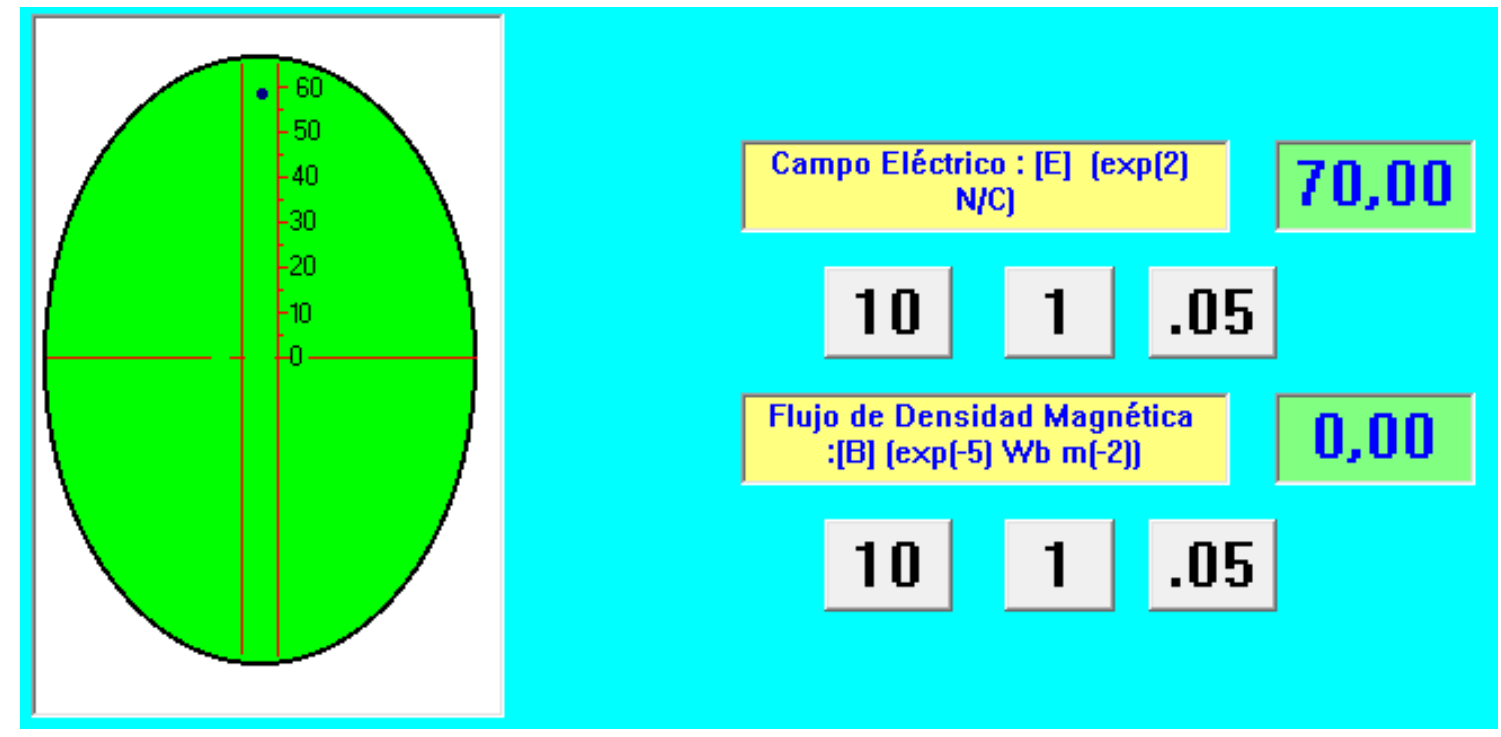

Figura 4.Vista de la pantalla al aplicar un campo eléctrico.

Manteniendo ese campo eléctrico constante, se modifica ahora el campo magnético hasta volver el haz a su trayectoria original, o sea sin desviación. Se registra el valor del campo magnético necesario. Se hacen interpolaciones si es necesario, a fin de disminuir los errores. Para ello se puede pulsar la tecla "Ver escala ampliada", como se ve en la Figura 5 para ajustar la interpolación sobre una pantalla más detallada que la anterior. 


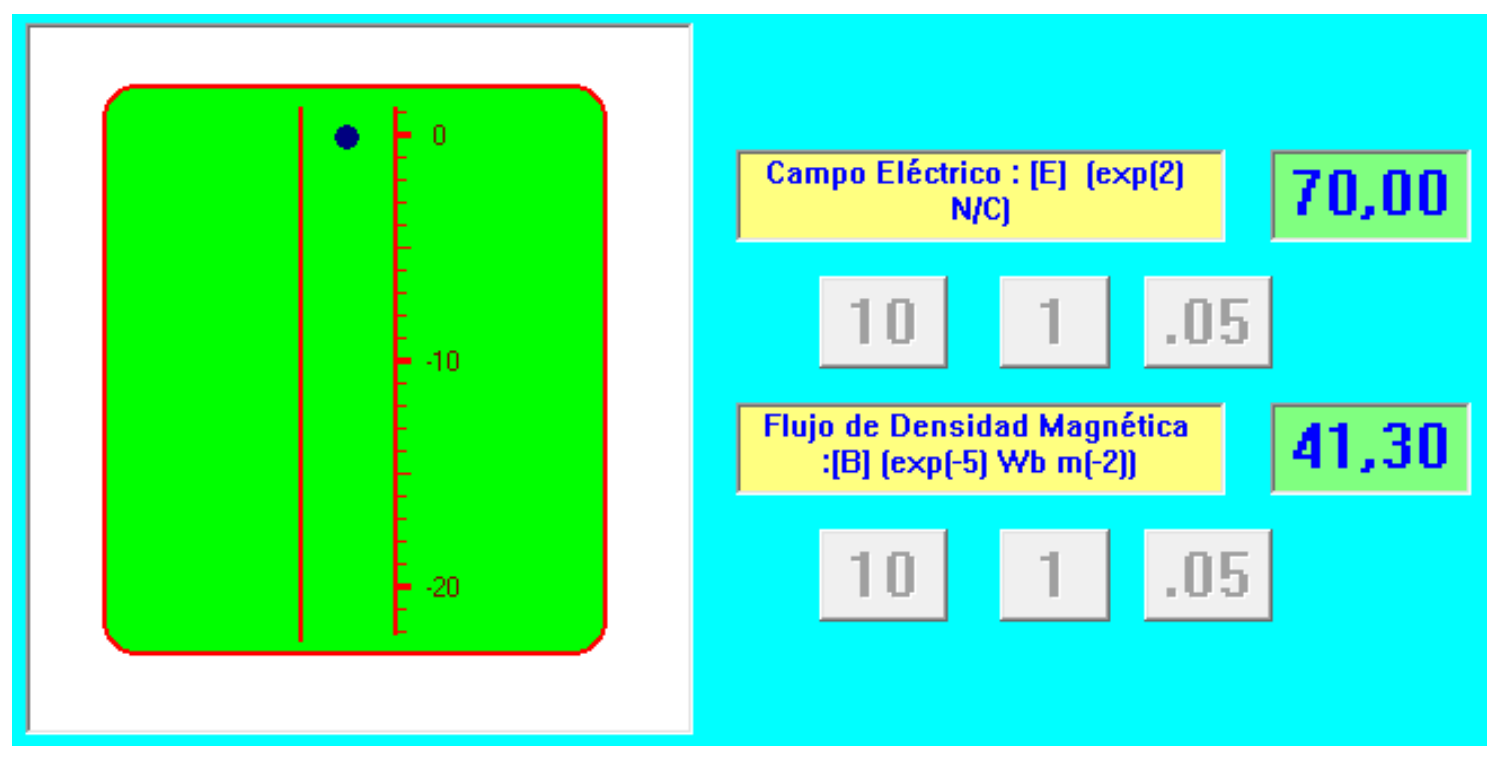

Figura 5. Vista de la pantalla con escala ampliada cuando se aplica B para llevar la desviación del haz a cero.

Como información adicional, se dan algunas características constructivas del tubo que se usa en la simulación. Se indica que el mismo tiene una longitud de placas $\ell$ (donde se aplica el campo eléctrico E) de $0,10 \mathrm{~m}$ y una distancia $x$ desde el final de las placas hasta la pantalla fluorescente de 0,092 m. En la Figura 6 puede verse más claramente la definición de estos parámetros del equipo. Se analizará la forma de realizar los cálculos. A través de las leyes de Newton se sabe que

$$
\begin{gathered}
\text { Fuerza }=m \times a \\
\text { espacio }=1 / 2 \times a \times t^{2}
\end{gathered}
$$

Cuando se aplican un campo eléctrico $(E)$ y uno magnético $(B)$ que anulan la desviación del haz de rayos catódicos, se verifica que las dos fuerzas son iguales y opuestas:

$$
B \times e \times v=e \times E
$$

de donde se puede calcular la velocidad de las partículas, como:

$$
v=\frac{E}{B}
$$


Cuando sólo se aplicó el campo eléctrico E, la partícula sufre una aceleración en la dirección perpendicular a las placas, que es la responsable de la desviación $\delta$ (Figura 6) y que se puede calcular a través de la ley de Newton:

$$
\begin{gathered}
e \times E=m \times a \\
a=e \times E / m
\end{gathered}
$$

La deflexión $\delta$ se puede relacionar con la aceleración y el tiempo $t$, que tarda la partícula en recorrer la longitud entre placas $\ell$

$$
\begin{gathered}
\delta=1 / 2 \times a \times t^{2} \\
t=\ell / v
\end{gathered}
$$

Combinando las últimas tres ecuaciones, se llega a:

$$
\delta=1 / 2 \frac{e \times E}{m} \times\left(\frac{\ell}{v}\right)^{2}
$$

y reemplazando la velocidad por su igual $v=\frac{E}{B}$ y reordenando, queda:

$$
\frac{e}{m}=\frac{2 \times \delta}{\ell^{2}} \times \frac{E}{B^{2}}
$$

Realizando algunos reemplazos de variables en la Ecuación 10 se puede llegar a otra expresión donde participan “ $d$ " $y$ “ $x$ ” (Figura 6).

$$
\frac{e}{m}=\frac{d \times v^{2}}{E \times\left(1 / 2 \times \ell^{2}+x \times \ell\right)}
$$

En este caso es necesario medir el desplazamiento $d$ sobre la pantalla luego de aplicar el campo eléctrico E. Las restantes variables de la ecuación son conocidas. El valor aceptado para la relación carga/masa es $1,76 \times 10^{11} \mathrm{C} / \mathrm{Kg}$. 


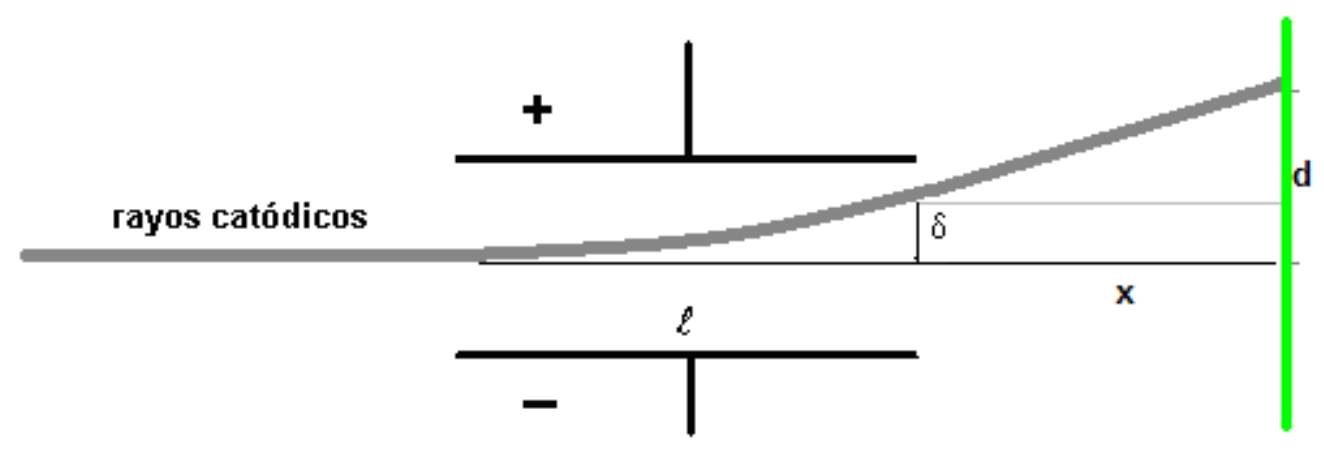

Figura 6. Parámetros del tubo de rayos catódicos.

Como se vio en el desarrollo anterior el alumno puede calcular los parámetros fundamentales de las partículas que componen los Rayos Catódicos. Para la mayoría de los alumnos iniciales de química el desarrollo matemático que se ha visto no es fácil, ya que deben recordar varias leyes de la física para llegar al resultado correcto. Es conveniente sugerir que recurran a la bibliografía, por ejemplo Sears, (1958), o al anexo que figura en la guía de trabajos prácticos, que se suministra al alumno, donde se muestran las relaciones que llevan al cálculo de e/m con mayor detalle.

\section{Simulación de la experiencia de Millikan y la determinación de la carga del electrón}

A través de la experiencia de Millikan es posible calcular la carga del electrón y entonces en base a la relación obtenida en la simulación anterior, se puede obtener la masa de la partícula.

En un medio viscoso como el aire, una gotita de aceite de un spray cae con velocidad constante y dada por la siguiente expresión:

$$
\begin{gathered}
v=(\text { fuerza gravitatoria }) /(\text { resistencia viscosa }) \\
\qquad v=\frac{m \times g}{6 \times \pi \times \mu \times r}
\end{gathered}
$$

donde $m$ : masa de la gota de aceite, $g$ : aceleración de la gravedad, $\mu$ : viscosidad del aire y $r$ : radio de la gota.

Por acción de la radiación $\mathrm{X}$, que ioniza moléculas que componen el aire, las gotitas se cargan negativamente y cuando se aplica el campo eléctrico comienzan a disminuir su velocidad de acuerdo con: 


$$
v^{\prime}=\frac{m \times g-q \times E}{6 \times \pi \times \mu \times r}
$$

llegando un momento que si el campo tiene intensidad suficiente, la gota se detiene y queda suspendida en el aire. En ese momento se verifica que $m \times g=q \times E$. Conociendo el radio de la gota, $r$, se tiene

$$
4 / 3 \times \pi \times r^{3} \times \delta \times g=q \times E
$$

de donde se puede despejar $q$, el valor de la carga de la gotita.

De la Ecuación 13 midiendo la velocidad, se puede calcular $r$ a través de los siguientes pasos:

$$
\begin{gathered}
v=\frac{4 / 3 \times \pi \times \delta \times g \times r^{3}}{6 \times \pi \times \mu \times r} \\
v=\frac{2 \times \delta \times g \times r^{2}}{9 \times \mu} \\
r^{2}=\frac{9 \times \mu \times v}{2 \times \delta \times g}
\end{gathered}
$$

Es necesario tener cuidado con el uso de las unidades. Las utilizadas por el programa son: $\mu:\left[\mathrm{N} \cdot \mathrm{s} \cdot \mathrm{m}^{-2}\right] ; v:[\mathrm{m} / \mathrm{s}] ; \delta:\left[\mathrm{Kg} \cdot \mathrm{m}^{-3}\right] ; g:\left[\mathrm{m} \cdot \mathrm{s}^{-2}\right]$, donde el Newton $\mathrm{N}=\mathrm{Kg} \cdot \mathrm{m} \cdot \mathrm{s}^{-2}$, y da como resultado el radio de la gotita en metros.

El valor para la densidad del aceite que toma el programa es $840 \mathrm{~K} / \mathrm{m}^{3}$, para la viscosidad del aire a $20{ }^{\circ} \mathrm{C} 1,82 \times 10^{-5} \mathrm{~N} \cdot \mathrm{s} \cdot \mathrm{m}^{-2}$, y para la aceleración de la gravedad 9,81 $\mathrm{m} \cdot \mathrm{s}^{-2}$.

En la Figura 7 se muestra el aparato de Millikan usado en la simulación, con una vista parcial de su interior. Se pueden observar el aparato para generar el spray de gotitas de aceite, las placas a través de las cuales se puede aplicar el campo eléctrico, el agujero de la placa superior por donde pasa la gotita a seleccionar, el tubo de rayos X para cargar las gotitas y el anteojo a través del cual se harán las mediciones. 


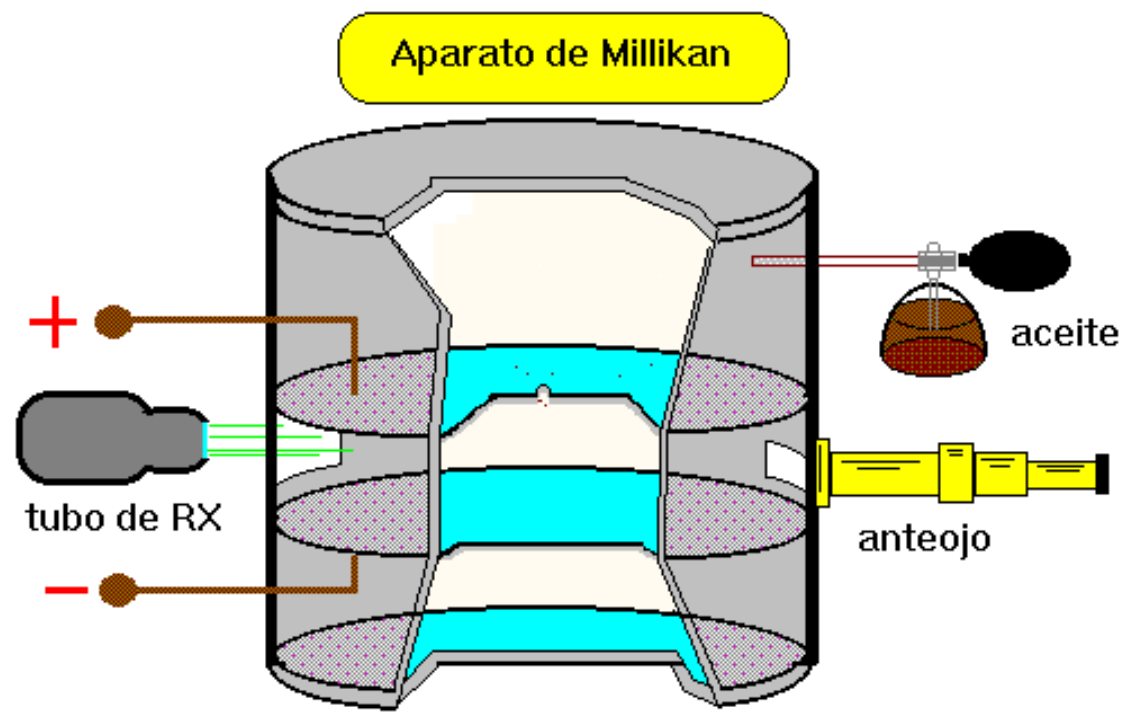

Figura 7. Esquema del aparato de Millikan utilizado en la simulación.

La secuencia de trabajo con el programa es producir un rocío de partículas de aceite y seleccionar una de las que pasan por la abertura de la placa superior. Se la sigue a través del campo visual de un anteojo, que está adosado al equipo. En la Figura 8 se muestra el campo visual del anteojo donde se ve la gotita cayendo. Es necesario medir la velocidad de caída de la partícula seleccionada, tomando el tiempo que tarda en recorrer una cierta distancia sobre la reglilla graduada. Rápidamente se aplica un campo eléctrico para frenar la caída de la partícula y evitar así que se pierda en el fondo del equipo. El campo eléctrico se regula con un ajuste fino a fin de mantener la gotita de aceite suspendida en el aire. El proceso se repite 6-7 veces con varias gotitas.

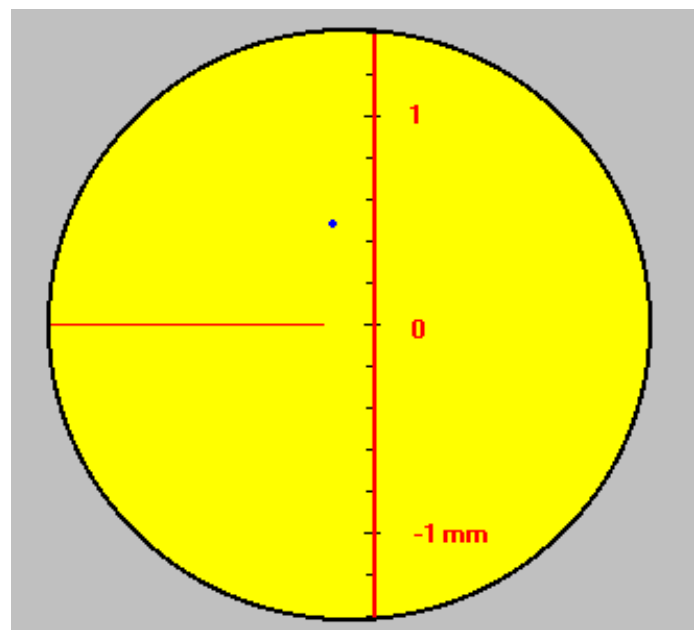

Figura 8. Campo visual del anteojo a través del cual se realizan las mediciones. 
El programa está diseñado para obtener en las gotitas cargas de 2, 3, 4 ó 5 unidades electrónicas. A partir de esta información el alumno debe encontrar que entre todos sus resultados hay un común divisor, el cual se postulará que es la carga del electrón.

Cada unidad de campo eléctrico aplicado en los botones representan $10^{4} \mathrm{~N} / \mathrm{C}$, por lo que el valor leído debe multiplicarse por ese número. Algunos resultados típicos obtenidos por los alumnos al ejecutar el programa pueden verse en la Tabla 1.

Tabla 1

Resultados típicos de varias experiencias del programa Millikan.

\begin{tabular}{ccccc}
\hline Experiencia & $\mathrm{r}(\mathrm{m})\left(\times 10^{5}\right)$ & $\mathrm{v}(\mathrm{m} / \mathrm{s})\left(\times 10^{5}\right)$ & $\mathrm{E}\left(\times 10^{-4}\right) \mathrm{N} / \mathrm{C}$ & $\mathrm{q}\left(\times 10^{19}\right) \mathrm{C}$ \\
\hline 1 & 0,118 & 14,10 & 7,22 & 7,9 \\
2 & 0,117 & 13,90 & 12,40 & 4,5 \\
3 & 0,074 & 5,55 & 4,40 & 3,2 \\
4 & 0,121 & 14,70 & 9,80 & 6,2 \\
5 & 0,103 & 10,60 & 4,80 & 7,7 \\
6 & 0,097 & 9,34 & 9,50 & 3,2 \\
7 & 0,088 & 7,81 & 2,97 & 7,9 \\
\hline
\end{tabular}

Buscando el máximo común divisor, que resulta ser $\cong 1,6 \times 10^{-19} \mathrm{C}$, se obtiene la carga del electrón. Los cocientes son 4,93;2,81;2;3,87;4,81;2;4,93. Estos cocientes corresponderían a 5, 3, 2, 4, 5, 2, 5 electrones. Con estos valores enteros la carga en cada caso daría: 1,$58 ; 1,5 ; 1,6 ; 1,55 ; 1,54 ; 1,6 ; 1,58 \times 10^{-19} \mathrm{C}$ lo cual coincide dentro de los errores experimentales con el valor aceptado.

Con este resultado y el de la simulación que se describió anteriormente, donde se obtenía la relación entre la carga y la masa del electrón, se calcula la masa de la partícula como: $9,1 \times 10^{-31} \mathrm{Kg}$.

Con estas 2 simulaciones que se han descrito con cierto detalle, es posible caracterizar al electrón completamente a través de su masa y de su carga. Ahora surge la pregunta al alumno de quién balancea esa carga negativa del electrón ya que la materia es eléctricamente neutra. En este momento se sugiere continuar con las otras simulaciones siguiendo una secuencia histórica a fin de que el alumno vaya viendo cual fue el aporte que cada una de ellas suministró al avance del modelo. Así el descubrimiento de la 
radiactividad, la experiencia de la lámina de oro de Rutherford, los rayos $\mathrm{X}$ característicos (postulación de número atómico) y la interpretación de los espectros electrónicos por Bohr figuran entre las más relevantes.

Los programas, además de ser utilizados en la ejecución de trabajos prácticos de química general, pueden ayudar al profesor en la clase teórica en la etapa de expansión de las ideas lo cual incentiva, de acuerdo a nuestra experiencia, la atención del alumno en el seguimiento de la clase (Baggio, 2010).

Parte de los programas también fueron presentados en distintos cursos y talleres para docentes en los últimos años recibiendo en general buena acogida por parte de los mismos. En el último de ellos, realizado en agosto de 2015, participaron cerca de treinta docentes entre profesores y estudiantes del profesorado (Baggio, 2015).

Al finalizar los cursos que se dictan para docentes y que contienen las simulaciones que aquí se describen, se les requiere que completen una brevísima encuesta que consta de 6 preguntas, con una ponderación de 1 a 5, de acuerdo al siguiente esquema:

Grados de acuerdo: 1 (Totalmente en desacuerdo); 2 (en desacuerdo); 3 (neutro); 4 (de acuerdo); 5 (Totalmente de acuerdo). Las preguntas son las siguientes.

1. Las simulaciones son útiles para los cursos iniciales de Química, ya que facilitan la compresión de los temas desarrollados.

2. Las simulaciones, como las utilizadas, resultan motivadoras para el alumno.

Respecto de la materia/s que Ud. dicta:

3. En el laboratorio: ¿considera que las simulaciones pueden ser usadas como un buen complemento de esas actividades?

4. ¿Considera que algunos de los laboratorios que desarrolla en su materia pueden ser reemplazados ventajosamente por simulaciones?

5. En sus clases teóricas. ¿Podría incorporar simulaciones pensando que esto redundaría en una mayor integración de la materia?

6. En las clases de problemas ¿Considera ventajoso en uso de guías digitales, como las presentadas en lugar de las clásicas impresas? 


\section{CONCLUSIONES}

Algunos de los programas (números 1 a 6) son utilizados por los alumnos en el curso de química general de la UNPSJB en el trabajo práctico de estructura de la materia. A través de esta actividad se puede "experimentar" en un tema abstracto para el cual la universidad no dispone del equipamiento correspondiente. La respuesta, en general, es positiva, ya que los alumnos son proclives al uso de las nuevas tecnologías y el uso de imágenes y animaciones les facilita la entrada a un tema de las características del modelo atómico. Los informes que presentan los alumnos, luego de realizada la actividad de simulación, muestran una razonable comprensión de los fenómenos y despiertan un entusiasmo que no siempre se logra con la mera lectura del libro de texto o la asistencia a la clase teórica.

En los que hace a los cursos y talleres dictados para docentes, en la encuesta que se les realiza y que se describió anteriormente, el puntaje promedio para las respuestas fue superior a 4, con un número mínimo de respuestas menores a 3, sobre un máximo de 5 puntos.

Una experiencia que recogió el autor a través del dictado de innumerables cursos para docentes con éstas y otras simulaciones, es que el entusiasmo que presentan éstos cuando comentan los resultados del mismo en las encuestas, se diluye cuando regresan a sus lugares de trabajo y en general les cuesta poner en marcha actividades con sus alumnos, particularmente en la enseñanza media.

El intercambio a través del correo electrónico o personal con ellos indica que muchos presentan excusas para utilizar las simulaciones, como falta de tiempo, demasiada cantidad de alumnos a cargo, dificultad en el cumplimiento de los programas, a pesar de sus comentarios favorables sobre las mismas. La opinión personal es que existe un cierto temor en muchos de ellos, al ver quizás la facilidad con la que los jóvenes alumnos manejan los ordenadores en contraposición con algunos docentes que se sienten aun inseguros en el manejo de las nuevas tecnologías.

En este caso es importante alentarlos, mencionando que son ellos quienes conocen la disciplina y tienen las herramientas didácticas y que la simplicidad en el manejo de los programas debería animarlos para utilizarlos en el aula. Cuando las condiciones de 
distancia lo han permitido, se ha participado en clases con algunos de estos docentes y sus alumnos, notándose en general una mayor predisposición en ellos al tener el soporte de quienes los han capacitado en el uso de los programas. Creemos que es una cuestión de tiempo para que la química también pueda sacar provecho del uso de los equipos de que disponen docentes y alumnos y que han sido entregados por las autoridades en varios países de la región en los últimos años.

A fin de que los programas puedan ser utilizados en escuelas y universidades de nuestros países, una copia de los instalables y las guías para los alumnos, se distribuye sin cargo para los docentes interesados que se lo soliciten al autor a través del correo electrónico. Así mismo, el autor se pone a disposición de quienes deseen utilizar los programas en sus cursos, para orientarlos frente a cualquier dificultad que se les pueda presentar.

\section{REFERENCIAS}

Atkins, P. y Jones, L. (1997). Chemistry: Molecules, Matter and Change.3ra Ed. (p.354). New York: W. H. Freeman \& Company.

Baggio, S. (2009). Simulaciones en un curso de química básica, como complemento de las actividades de aula y laboratorio, con un enfoque de investigación guiada. Educación en la Química. 15(1), 41-53.

Baggio, S. (2010). Las simulaciones como una forma de efectivizar la unidad teóricopráctica en un curso de química general. Educación en la Química. 16(2), 109-20.

Baggio, S. (2015). Reunión de Educadores en Química de la República Argentina, REQ XVII, Universidad Nacional del Chaco Austral, Presidencia R. S. Peña, Chaco, agosto $12-15$.

Babor, J. A. y Ibarz, J. (1968). Química general moderna (p.157-160). Barcelona: Manuel Marín y Cia.

de Jong, T. (2011). Instruction based on computer simulations. En Mayer, R.E. y P.A. Alexander (Eds.), Handbook on Research on Learning and Instruction (pp.446-466). New York: Routledge.

Farrell, J. J., Spencer, J. N. y Moog, R. S. (1999). A guided inquiry general chemistry course. Journal of Chemical Education. 76(4), 570-574. 
Garrett, A. B. (1962a). X-Rays: W. C. Roentgen. Journal of Chemical Education. 39(7), 360-361.

Garrett, A. B. (1962b). Radioactivity: Henri Becquerel. Journal of Chemical Education. 39(10), 533-534.

Gillespie, R. J. (1991). What is wrong with the general chemistry course? Journal of Chemical Education.68(3).192-194.

Gillespie, R. J., Spencer J. N. y Moog, R. S. (1996). Demystifying introductory chemistry. Part 1. Electron configuration from experiment. Journal of Chemical Education.73(7).617-622.

Haigh, C. W. (1995). Moseley's Work on X-Rays and Atomic Number. Journal of Chemical Education. 72(11), 1012-14.

Herron, J. D. (1977). Rutherford and the nuclear atom. Journal of Chemical Education. 54(8), 499.

Herzberg, G. (1944). Atomic Spectra and Atomic Structure (p.11). New York: Dover Publications, Inc.

Karplus, R. (1977). Science teaching and the development of reasoning, Journal of Research in Science Teaching. 14(2), 169-175.

Kirschner, P.A., Sweller, J., \& Clark, R.E. (2006). Why minimally guided instruction does not work. Educational Psychologist, 41, 2, 75-86.

Kolb D.A. (1984). Experiential Learning experience as a source of learning and development. New Jersey: Prentice Hall.

Mahan, B. M. y Myers, R. J. (1987). University Chemistry, 4ta Ed. (p.505). California. The Benjamin/Cummings Publishing Company, Inc.

Mayer, R.E. (2009). Multimedia learning, 2da Ed. New York: Cambridge University Press.

Moog, R. S. y Farrell, J. J. (2004). Química: Un enfoque inquisitivo. 2da Ed. (pp.1-55). USA: John Wiley and Sons, Inc.

Papert, S. (1980). Mindstorms: Children, computers, and powerful ideas. New York: Basic Books.

Pauling, L. (1984). View from the top: The beginning course in chemistry .Chemtech, june, 326-327. 
Peake, Barrie M. (1989). The discovery of electron, proton and neutron. Journal of Chemical Education. 66(9), 738.

Pearson, E.F. (2005), Revisiting Millikan's oil-drop experiment. Journal of Chemical Education. 82(6), 851-853.

Rieber, L.P. (2005). Multimedia learning in games, simulations, and microworlds. En: Mayer, R.E. (Ed), The Cambridge Handbook of Multimedia Learning. (pp.549-567). New York: Cambridge University Press.

Sears, F. W. (1958). Electricidad y Magnetismo (p.228). Madrid: Aguilar S.A. de Ediciones, Madrid.

Tobias, S., y T.M. Duffy (Eds.). (2009). Constructivist instruction. Success or failure? New York: Routledge. 